\title{
Demonstrator influence on observer diet preference: Effects of simple exposure and the presence of a demonstrator
}

\author{
BENNETT G. GALEF, JR., DEBORAH J. KENNETT, and MONI STEIN \\ McMaster University, Hamilton, Ontario, Canada
}

\begin{abstract}
Previous studies in our laboratory have demonstrated that a naive rat (an observer), after interacting briefly with a previously fed conspecific (a demonstrator), will exhibit an enhanced preference for the diet its demonstrator had been fed. The present studies were undertaken to determine whether demonstrator-induced alterations in observer diet preference were the result of simple exposure of observers to diet-identifying cues emitted by demonstrators during the period of demonstrator-observer interaction. Our results indicated that observer experience of diet-related cues in the stimulus context provided by the presence of a demonstrator was sufficient to enhance observer preference for a diet, whereas simple exposure to that diet was not. We concluded that demonstrator influence on observer diet preferences was not the consequence of simple exposure of observers to demonstrator-emitted cues reflecting demonstrators' diet.
\end{abstract}

The interaction of an observer rat with a recently fed conspecific demonstrator substantially enhances the observer's subsequent preference for whatever diet its demonstrator has eaten (Galef, 1983; Galef \& Wigmore, 1983; Posadas-Andrews \& Roper, 1983). In experiments previously conducted in our laboratory, food-deprived individual rats (demonstrators) were fed either cocoa- or cinnamon-flavored diet for $30 \mathrm{~min}$, and each demonstrator was then allowed to interact with an experimentally naive rat (an observer) for $15 \mathrm{~min}$. Observers, when subsequently offered a choice between cinnamon- and cocoaflavored diets, exhibited a robust preference for whichever diet their respective demonstrator had eaten. Analysis of the conditions under which demonstrators could influence the future diet preference of observers indicated that olfactory cues passing from demonstrator to observer were sufficient to modify observers' subsequent diet preference (Galef \& Wigmore, 1983).

The series of studies reported below were undertaken to investigate the behavioral processes responsible for demonstrator-induced changes in observer diet preference. It is, for example, possible that the effective olfactory cue passing from demonstrator to observer is simply the smell of the food a demonstrator has contacted and ingested.

\footnotetext{
The research reported here was supported by Natural Sciences and Engineering Research Board of Canada Grant AP307 and a McMaster University Research Board grant to B.G.G. Jr. We thank Sandra Vegeris, Tania Masing, and Roy Kaufmann for technical assistance and Mertice Clark and Harvey Weingarten for their critical readings of earlier drafts of this manuscript. D. J. Kennett is now at Brock University, St. Catharines, Ontario. Moni Stein is now at the Medical School of the University of Toronto. Requests for reprints should be sent to Bennett G. Galef, Jr., Department of Psychology, McMaster University, Hamilton, Ontario, Canada L8S 4K1.
}

Exposure to such demonstrator-emitted food odors might result in increased observer familiarity with a demonstrator's diet and consequent enhanced observer acceptance of that diet (Leon, Galef, \& Behse, 1977; Siegel, 1974). In this case, one could explain the influence of demonstrators on observers as a simple familiarity effect (Hill, 1978).

Alternatively, it might be that the demonstrator-emitted cue that is effective in altering the diet preference of an observer is a combination of the smell of demonstratoringested diet and some demonstrator-produced signal. If such were the case, an explanation of demonstrator effects on observer diet preference in terms of simple exposure to or familiarity with demonstrators' diets would be misleading. Determining whether simple exposure effects can account for demonstrator influence on observer diet preference is a necessary first step in analysis of the behavioral mechanisms underlying transmission among rats of information concerning distant diets.

In the series of experiments described below, we used two complementary approaches to determine whether demonstrator-induced simple familiarity with a diet was an adequate explanation for the observed effects of demonstrators on observers' diet preferences. In Experiment 1, we first made observers familiar with two diets, then allowed each observer to interact with a demonstrator that had eaten one of those two diets, and subsequently allowed each observer to choose again between the two familiar diets, one of which had been eaten by its demonstrator. In Experiments 2 and 4, we directly examined the effects of observer exposure to a novel diet on subsequent observer preference for that diet in a choice situation. In both types of experiment, the outcome was inconsistent with an explanation of demonstrator influence on observer diet preference in terms of the effects of simple familiarity. 


\section{EXPERIMENT 1}

As discussed above, it is possible that observer interaction with a demonstrator affects subsequent observer diet preference simply by making the diet eaten by a demonstrator familiar to the observer and reducing the observer's subsequent neophobic response to that diet. If this is the case, observers choosing between two familiar diets should exhibit markedly attenuated effects of interaction with a demonstrator when choosing between familiar rather than novel diets. Observers with extensive previous experience with two diets to be offered in a preference test should be thoroughly familiar with both. Any additional familiarity with one of the test diets, resulting from a brief period of observer interaction with a demonstrator fed that diet, should be overwhelmed by the observer's extensive experience with both diets. Hence, examination of demonstrator influence on the diet preference of observers choosing between familiar diets provides an indirect test of the adequacy of simple exposure effects to explain demonstrator influence on observer diet preference.

\section{Method}

Subjects. Thirty-two 42-day-old Long-Evans rats born in the McMaster colony to breeding stock acquired from Blue Spruce Farms (Altamont, NY), served as observers in the procedure described below. Thirty-two additional 60-day-old rats from the same source served as demonstrators.

Apparatus. The observers were housed singly and tested in $22 \times$ $24 \times 27.5 \mathrm{~cm}$ wire-mesh hanging cages (Wakemann Co., Baltimore, Maryland.)

Procedure. Treatment of subjects during the experiment was as follows (see Figure 1).

(1) Individual observers were placed in the apparatus and assigned randomly to one of two preexposure conditions. Those observers assigned to the $\mathrm{Cin} / \mathrm{Coc}$ preexposure group were allowed to feed ad lib for 2 days from two food cups, one containing powdered Purina Laboratory Rodent Chow adulterated $2 \%$ by weight with Hershey's Pure Cocoa (Coc) and one containing powdered Purina Laboratory Rodent Chow adulterated $1 \%$ by weight with McCormick's Fancy Ground Cinnamon (Cin). The subjects assigned to the control preexposure group were treated identically to those assigned to the Cin/Coc preexposure group, except that they had access for 2 days to two food cups containing unadulterated powdered Purina Laboratory Rodent Chow.

(2) Each of 32 23-h food-deprived demonstrator rats was offered either $\mathrm{Coc}$ or Cin for $30 \mathrm{~min}$.
(3) Each observer was allowed to interact for $15 \mathrm{~min}$ with a demonstrator of the same sex. Demonstrators presented to half the observers in both the Cin/Coc and control preexposure groups had previously eaten the Coc diet. Demonstrators presented to the remainder of the observers in each preexposure group had previously eaten the Cin diet.

(4) Demonstrators were removed from the experiment, and each observer was offered, for $221 / 2 \mathrm{~h}$ a choice between fresh samples of $\mathrm{Coc}$ and $\mathrm{Cin}$. The experimenter determined intake of each diet 3,7 , and $22 \frac{1}{2} \mathrm{~h}$ after initiation of observer testing.

\section{Results and Discussion}

The main results of Experiment 1 are presented in Figure 2, which shows, as a percentage of total intake, the mean amount of Coc diet eaten by observers in the $\mathrm{Cin} / \mathrm{Coc}$ and control preexposure groups whose demonstrators had eaten either the Coc or the Cin diet. As is evident from inspection of the figure and as statistical tests confirmed, the diet eaten by demonstrators profoundly affected the food choices of observers in both the Cin/Coc and the control preexposure groups (Mann-Whitney $U$ tests, see Figure 2 for $p$ values). Observers ate an average of $2.2 \pm .2 \mathrm{~g}$ of diet during the first $3 \mathrm{~h}$ of testing, $3.6 \pm .2 \mathrm{~g}$ during the first $7 \mathrm{~h}$ of testing, and $23.0 \pm .8 \mathrm{~g}$ during the entire $22^{1 / 2}$-h test period. Although preexposure of observers to the test diets (Cin/Coc preexposure group) reduced the duration of the effects of demonstrators' diet on observers' food preferences, the initial diet-preference influence of interaction with a demonstrator was not reduced by 2 days' preexposure to the test diets. This finding renders unlikely the interpretation that the effects of demonstrators on observers' diet preferences result solely from familiarity with the taste or smell of a diet experienced during $15 \mathrm{~min}$ of interaction with a demonstrator. The more rapid waning of observers' preferences for their respective demonstrators' diets in the Cin/Coc- than in the control-preexposure group does, however, suggest that diet novelty may play a role in the maintenance of observer preference for demonstrators' diets.

\section{EXPERIMENT 2}

If demonstrator-induced familiarity with one of the test diets were responsible for the influence of demonstrators on observers' diet preferences, one would expect that the

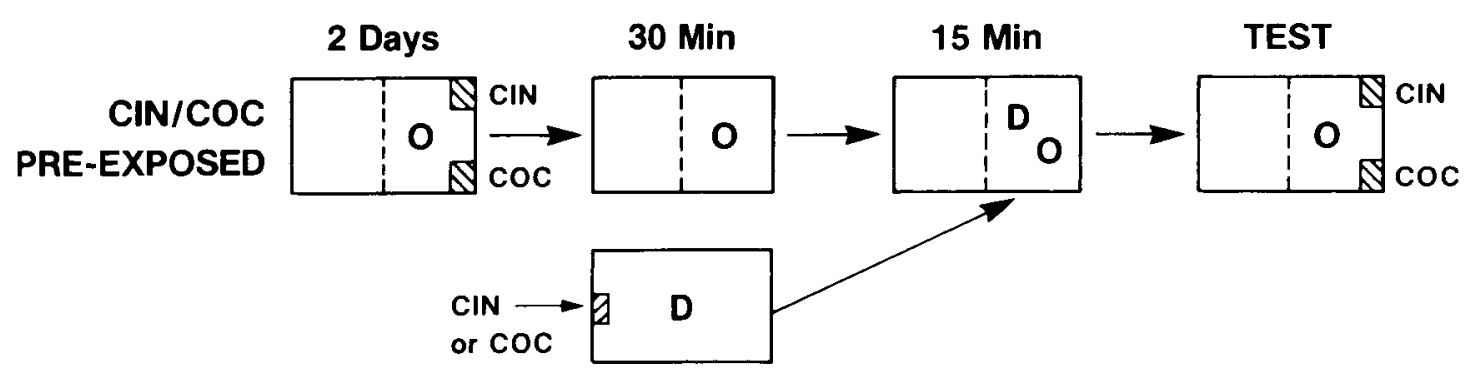

\section{$\begin{array}{llll}\text { STEP } 1 & \text { STEP } 2 & \text { STEP } 3 & \text { STEP } 4\end{array}$}

Figure 1. Schematic diagram of the procedures of Experiment $1(0=$ observer; $D=$ demonstrator $)$. 


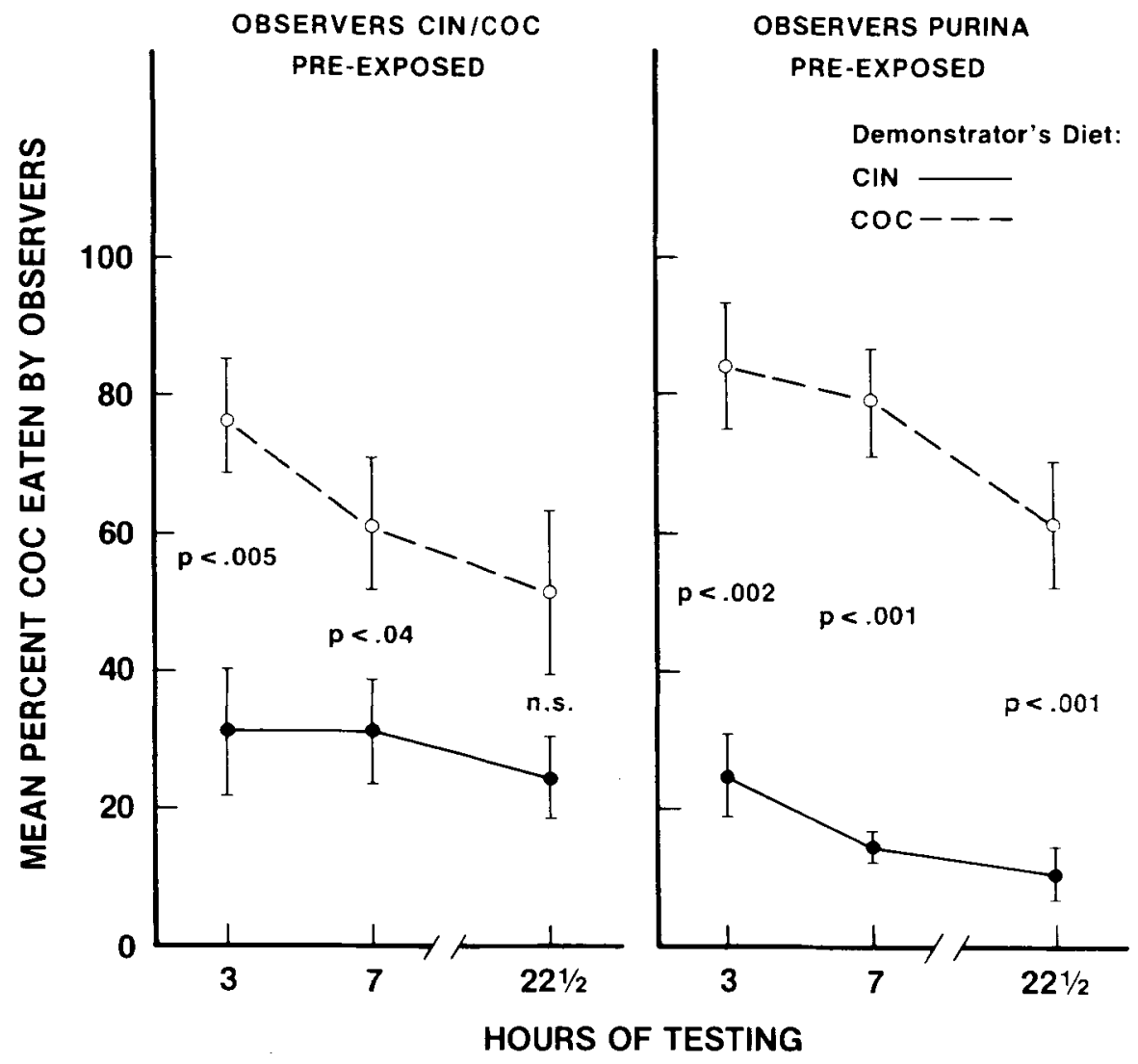

Figure 2. Mean amount of the Coc diet ingested, as a percentage of total amount eaten, by observers whose demonstrators had eaten either Cin or Coc. Bars indicate \pm 1 SE.

effect on observers of the interaction with demonstrators could be mimicked by directly preexposing each observer to one of the two diets to be used in testing.

\section{Method}

Subjects. Thirty-three 42-day-old Long-Evans rats from the McMaster colony served as subjects. All subjects had been maintained in same-sex groups of 3 to 5 littermates on ad lib Purina Laboratory Rodent Chow pellets and water from weaning at 21 days of age.

Procedure. Each subject was placed in a $22 \times 24 \times 27.5 \mathrm{~cm}$ wire-mesh hanging cage and allowed $24 \mathrm{~h}$ to become familiar with the apparatus. During this period, the subjects continued to be maintained on Purina Laboratory Rodent Chow pellets. At the end of this 24-h familiarization period, 11 subjects were assigned randomly to each of three diet preexposure groups. Immediately following group assignment, pellets of chow were removed from each subject's cage and each was offered, for $24 \mathrm{~h}$, unadulterated powdered Purina Laboratory Rodent Chow (Pur preexposure group), powdered Purina Laboratory Rodent Chow adulterated $2 \%$ by weight with Hershey's Pure Cocoa (Coc preexposure group), or powdered Purina Laboratory Rodent Chow adulterated $1 \%$ by weight with McCormick's Fancy Ground cinnamon (Cin preexposure group).

At the end of the 24-h period of diet preexposure, each subject was offered two food cups, one containing Cin and the other Coc. The experimenter weighed both food cups 3,6 , and $22 \mathrm{~h}$ after initiation of the diet preference test.

\section{Results and Discussion}

The main results of Experiment 2 are presented in
Figure 3, which shows the amount of the Coc diet, as a percentage of total amount eaten, ingested during preference testing by subjects in the Pur, Cin, and Coc preexposure groups. As can be seen in the figure, subjects in the latter two groups did not exhibit an enhanced preference for the diet to which they had been exposed for $24 \mathrm{~h}$.

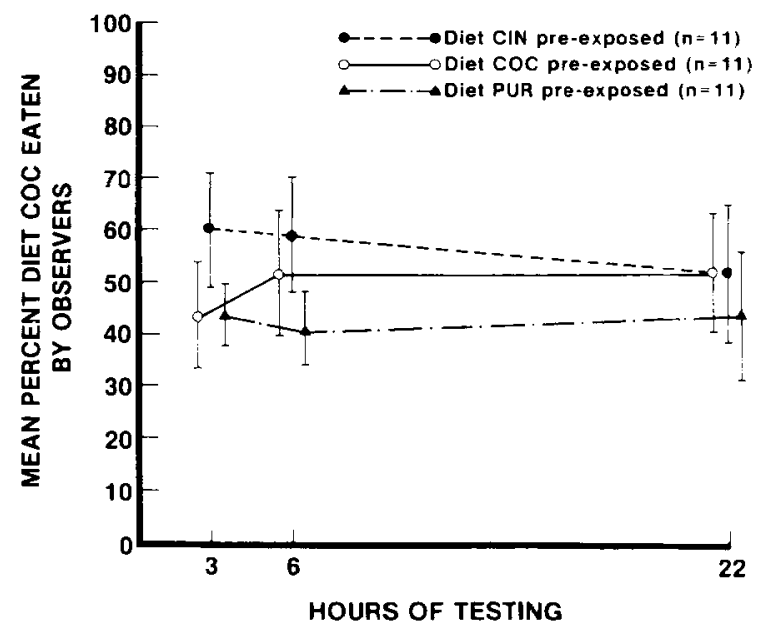

Figure 3. Mean amount of Coc ingested, as a percentage of total amount eaten, by subjects fed the Cin, Coc, or Pur diet for $24 \mathrm{~h}$. Bars indicate \pm 1 SE. 
To the contrary, comparison of the diet selections of subjects in the Cin and Coc preexposure groups revealed a tendency in both the 3- and 6-h measures for the subjects to exhibit a slightly enhanced preference for the unfamiliar test diet, although this tendency did not approach statistical significance (Mann-Whitney U tests, both U's $=66$, both ps n.s.). Observers ate an average of $2.2 \pm .3 \mathrm{~g}$ during the first $3 \mathrm{~h}$ of testing, $3.3 \pm .3 \mathrm{~g}$ during the first $7 \mathrm{~h}$ of testing, and $21.5 \pm .8 \mathrm{~g}$ during the entire $22 \mathrm{~h}$ test period.

We also examined the effects of $15 \mathrm{~min}$, rather than $24 \mathrm{~h}$, of observer exposure to either the Cin or the Coc diet on subsequent relative preference for those diets. Once again, simple exposure to a diet failed to result in enhanced observer preference for the diet to which observers were preexposed. During a 22-h test of observer preference between the Coc and Cin diets, observers $(\mathrm{n}=9)$ exposed to the Coc diet for 15 min ingested an average of $44.4 \%$ of the Coc $(\mathrm{SE}=10.0)$, and observers $(\mathrm{n}=9)$ exposed to the $\mathrm{Cin}$ diet for 15 min ingested an average of $44.0 \%$ of the Coc diet ( $S E=10.1$; Mann Whitney $U=33, p=$ n.s.).

The data of the present experiment, like those of Experiment 1 , are not consistent with the hypothesis that simple exposure of a subject to the Coc or Cin diet enhances subsequent subject preference for that diet.

\section{EXPERIMENT 3}

The results of Experiments 1 and 2 suggest that simple observer exposure to demonstrators' diets is not in itself sufficient to account for demonstrator influence on observer diet selection. It must therefore be that a demonstrator rat provides a context within which exposure to cues related to its diet renders that diet attractive to an observer. Exploration of the critical aspects of the context provided by the presence of a demonstrator clearly requires greater control than we had previously achieved over the stimulus complex that demonstrators present to observers. In the present experiment, we established the validity of a procedure permitting such control.

\section{Method}

Subjects. Thirty-two 42-day-old Long-Evans rats born in the McMaster colony served as observers in the procedures described below. Thirty-two additional 60 -day-old rats from the same source served as demonstrators.

Apparatus. During the experiment, all subjects were housed and tested as demonstrator-observer pairs in $42.5 \times 24 \times 27.5 \mathrm{~cm}$ wiremesh hanging cages (Wahmann, Co., Baltimore, MD). Each hanging cage was divided in two equal parts by a $1.25-\mathrm{cm}$ (1/2-in.) wiremesh partition $(24 \times 27.5 \mathrm{~cm})$ attached at the midpoints of each cage's $42.5-\mathrm{cm}$ sides.

During the period of interaction between observer and demonstrator (see Step 4 of Procedure below), subjects in the experimental group were placed in the apparatus illustrated in Figure 4. This apparatus was constructed from a $2.45-$ liter $(15.2 \mathrm{~cm}$ high, $19.0 \mathrm{~cm}$ top diameter, $14.0 \mathrm{~cm}$ bottom diameter) cardboard bucket (LilyTulip Inc., Toledo, Ohio) of the type commonly used by fast-food franchises.
A circular opening $5 \mathrm{~cm}$ in diameter was cut in the bucket wail $12 \mathrm{~cm}$ above the bucket floor. Through this hole was inserted, for half its length, a tube of .63-cm (1/4-in.) hardware-cloth, $16 \mathrm{~cm}$ long and $5 \mathrm{~cm}$ in diameter. The end of the tube inside the bucket was closed with hardware cloth; the end outside the bucket was left open. A cardboard lid served to prevent observers from leaving the bucket.

Procedure. Treatment of subjects assigned to the control group was as follows:

(1) Demonstrator and observer were placed in the apparatus on opposite sides of the partition and maintained with ad-lib access to Purina Laboratory Rodent Chow pellets for a 2-day period of familiarization.

(2) Demonstrator and observer were food deprived for $24 \mathrm{~h}$.

(3) The demonstrator was moved to an enclosure in a separate room and allowed to feed for $30 \mathrm{~min}$ on either the Cin or the Coc diet (see Step 1 of Procedure of Experiment 1 for composition of diets).

(4) The demonstrator was returned to its side of the apparatus and allowed to interact with its observer through the partition for $30 \mathrm{~min}$.

(5) The demonstrator was removed from the experiment and the observer was offered, for $22 \mathrm{~h}$, two weighed food cups, one containing Cin and one containing Coc.

Subjects in the experimental group were treated identically to those in the control group except during Step 4 of the above procedure. Immediately following feeding (Step 3), demonstrators in the experimental group were anesthetized (intraperitoneal injection with $50 \mathrm{mg} / \mathrm{kg}$ of sodium pentobarbital) and placed in the wire-mesh cylinder of the apparatus illustrated in Figure 4.

Observers were placed in the body of this apparatus to interact with the anesthetized demonstrator for $30 \mathrm{~min}$. At the end of the period of interaction, each observer in the experimental group was returned to its original cage and Step 5 of the procedure was initiated.

\section{Results and Discussion}

The main results of Experiment 3 are presented in Figure 5, which shows the mean amount of Coc eaten, as a percentage of total intake, by observers whose demonstrators ate either Coc or Cin. As is evident from inspection of the figure and as statistical tests confirmed (MannWhitney $U$ tests, see Figure 5 for $U$ and $p$ values), observers in both experimental and control groups exhibited an enhanced preference for the diet their respective

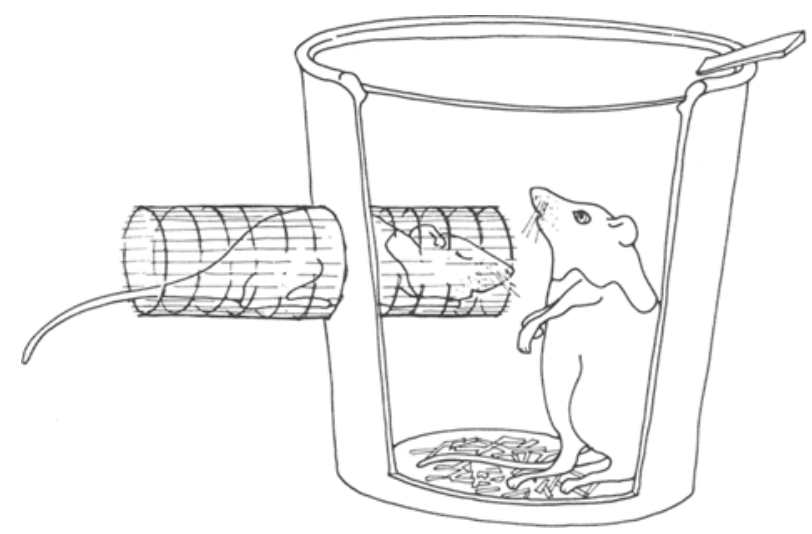

Figure 4. Apparatus in which the interaction of demonstrators and observers took place. 


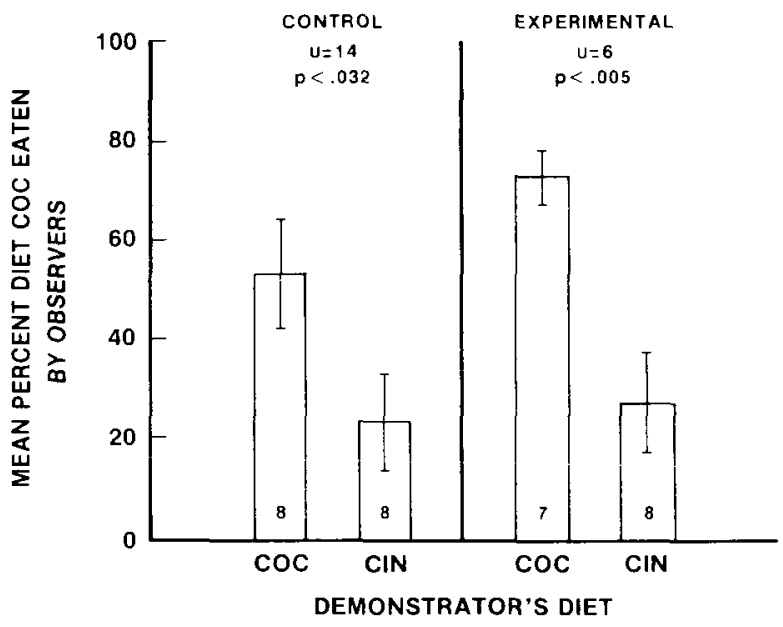

Figure 5. Mean amount of Coc ingested, as a percentage of total amount eaten, by observers whose demonstrators had eaten either Cin or Coc. Bars indicate \pm 1 SE; numbers in histograms = ns.

demonstrators ate. The present results demonstrate the adequacy of interaction between an anesthetized demonstrator and observer, in the apparatus illustrated in Figure 4, for communication of diet information from demonstrator to observer.

\section{EXPERIMENT 4}

If, as the results of Experiments 1 and 2 suggest, a demonstrator rat provides a context within which exposure to the smell of a demonstrator's diet influences the observers' subsequent diet selection, our problem is to define the nature of that context. Two very different possibilities suggest themselves. First, it is possible that the process of ingestion and digestion of a diet by a demonstrator alters that diet so as to make its smell or taste attractive to observers. Second, it is possible that the simple co-occurrence of a demonstrator rat and diet is sufficient to enhance subsequent diet preference in observers. In the present experiment, we excluded the first possibility.

\section{Method}

Subjects. Thirty-two 42-day-old Long-Evans rats obtained from Blue Spruce Farms (Altamont, NY) served as observers, and an additional 16 60-day-old rats from the same source served as demonstrators.

Apparatus. The apparatus was that described in the Method section of Experiment 3 .

Procedure. The procedure was similar to that described in the Method section of Experiment 3 except for treatment of the demonstrators with which observers interacted during Step 4 of Procedure. Each observer in the experimental group of the present experiment interacted with an anesthetized, food-deprived demonstrator that had not been fed prior to placement in the apparatus. Instead, following anesthetization, the head of each demonstrator was rolled in a bowl containing either Cin or Coc. The result was a demonstrator that had not ingested either Cin or Coc but whose face was powdered with one of them.
Observers in the control group were treated identically to those in the experimental group except that each observer interacted in the apparatus with a rat surrogate rather than a rat demonstrator. A surrogate was constructed by wrapping a rat-sized piece of cottonbatting in surgical gauze and rolling one end of the resulting cylindrical surrogate in either Cin or Coc. The diet-coated end of the surrogate was then placed in the same location in the apparatus as the heads of demonstrators exposed to observers in the control group.

\section{Results and Discussion}

The main results of Experiment 4 are presented in Figure 6, which shows the mean amount of Coc eaten, as a percentage of total intake, by observers whose demonstrators (or surrogate demonstrators) had been coated with either Cin or Coc.

As can be seen in Figure 6, observers that interacted with a diet-coated rat demonstrator exhibited a robust preference for the diet with which that rat demonstrator was coated. On the other hand, observers in the experimental group, which had interacted with a surrogate diet-coated demonstrator, failed to exhibit a preference for the diet with which that surrogate demonstrator was coated (Mann-Whitney $U$ tests, see Figure 5 for $U$ and $p$ values).

The results of the present experiment demonstrate, as do the results of Experiments 1 and 2, that simple exposure of observers to a diet is not sufficient to induce a preference for that diet in observers. Furthermore, the results of the present study indicate that ingestion of a diet by demonstrator rats is not a necessary condition for demonstrator influence on observer diet preference. Observer experience of an uningested diet in the context of a demonstrator rat suffices to alter subsequent observer preference for that diet.

The fact that observers prefer diets that are experienced on the surface of a rat to those experienced on a gauzewrapped piece of cotton batting suggests that it is the context in which a diet is experienced by an observer that

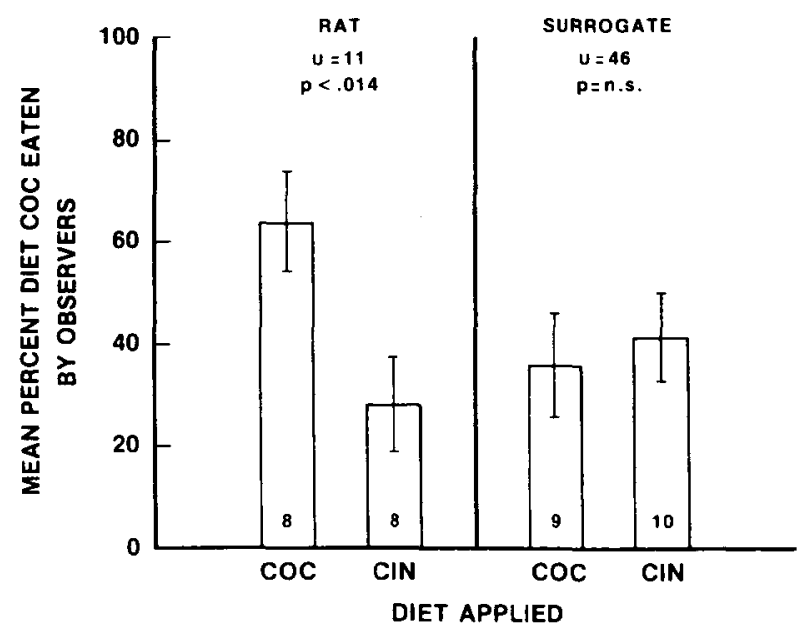

Figure 6. Mean amount of Coc ingested, as a percentage of total amount eaten, by observers exposed to surrogates or rats in Experiment 4. Bars indicate $\pm 1 \mathrm{SE}$; numbers in histograms $=$ ns. 
determines whether that diet will be preferred in future. The task remaining before us is to define the nature of the cues provided by a demonstrator rat which create a context within which diet exposure has effects on observer diet preference.

\section{GENERAL DISCUSSION}

The results of the present series of experiments strongly suggest that simple familiarity with the smell or taste of a diet is not sufficient to explain demonstrator-induced preference for a diet. Strupp and Levitsky (1984, Experiment 2), using a paradigm similar in many respects to ours, have also found that simple exposure of a rat to a diet does not enhance preference for that diet, whereas exposure to the same diet in the presence of a demonstrator markedly enhances preference for it. However, the present results cannot be interpreted as indicating that the effects of simple familiarity could not, in other circumstances, be responsible for enhanced diet preference. Posadas-Andrews and Roper (1983) have presented data that they interpreted as indicating that rats exposed to a swab containing almond extract subsequently exhibit enhanced preference for almond-extract-flavored food. Leon et al. (1977) found that prolonged exposure to peppermint extract enhances rat pup preference for peppermintextract-flavored food. Bronstein and Crockett (1976) reported that exposure to garlic odor enhances the preference of rat pups for a garlic-flavored diet. Clearly, there are circumstances in which simple exposure to characteristics of a diet enhances preference for it.

Domjan $(1976,1977)$ has suggested that single-stimulus procedures are more effective than choice procedures in revealing the role of diet novelty and familiarity in preference determination. Our failure to find simple exposure effects on diet preference may, therefore, simply reflect the test procedure we used in our experiments. Finding powerful demonstrator-induced alterations in observer diet preference in a test situation that attenuates effects of familiarity adds strength to the argument that the robust demonstrator influence on observer diet preference we observed did not depend on demonstrator-induced familiarity.

Taken together, the results of the present studies indicate that in circumstances in which simple exposure to a diet is not sufficient to alter subsequent diet preference in rats, a second, related phenomenon can be observed. Exposure to a diet in the stimulus context provided by the presence of a conspecific can enhance preference for a diet, even when simple exposure to that diet cannot.

\section{REFERENCES}

Bronstein, P. M., \& Crockett, D. P. (1976). Exposure to the odor of food determines the eating preference of rat pups. Behavioral Biology, 18, 387-392.

Domjan, M. (1976). Determinants of the enhancement of flavored water intake by prior exposure. Journal of Experimental Psychology: Animal Behavior Processes, 2, 17-27.

Domjan, M. (1977). Attenuation and enhancement of neophobia for edible substances. In L. M. Barker, M. R. Best, \& M. Domjan (Eds.), Learning mechanisms in food selection. Waco, TX: Baylor University Press.

GALEF, B. G., JR. (1983). Utilization by Norway rats ( $R$. norvegicus) of multiple messages concerning distant foods. Joumal of Comparative Psychology, 97, 364-371.

GALEF, B. G., JR., \& WIGMORE, S. W. (1983). Transfer of information concerning distant foods: A laboratory investigation of the "information centre" hypothesis. Animal Behaviour, 31, 748-758.

HiLl, W. F. (1978). Effects of mere exposure on preferences in nonhuman mammals. Psychological Bulletin, 85, 1177-1198.

Leon, M., Galef, B. G., JR., \& BeHSE, J. H. (1977). Establishment of pheromonal bonds and diet choice in young rats by odor preexposure. Physiology \& Behavior, 18, 387-391.

Posadas-ANDrews, A. , \& Roper, T. J. (1983). Social transmission of food preferences in adult rats. Animal Behaviour, 31, 265-271.

SIEGEL, S. (1974). Flavor preexposure and "learned safety." Journal of Comparative and Physiological Psychology, 87, 1073-1082.

STruPP, B. J., \& LeVITSKY, D. A. (1984). Social transmission of food preferences in adult hooded rats (Rattus norvegicus). Journal of Comparative Psychology, 98, 257-266.

(Manuscript received July 10, 1984; revision accepted for publication January 11, 1985.) 